\title{
Equivalence Verification of Polynomial Datapaths Using Ideal Membership Testing
}

\author{
Namrata Shekhar, Student Member, IEEE, Priyank Kalla, Member, IEEE, and Florian Enescu
}

\begin{abstract}
This paper addresses the problem of equivalence verification of RTL descriptions that implement arithmetic computations (such as ADD, MULT) over bit-vectors with finite widths. A bit-vector of size $m$ represents integer values from 0 to $2^{m}-1$; implying that the corresponding integer values are reduced modulo $2^{m}\left(\% 2^{m}\right)$. This suggests that bit-vector arithmetic can be efficiently modeled as algebra over finite integer rings, where the bit-vector size $(\mathrm{m})$ dictates the cardinality of the ring $\left(Z_{2^{m}}\right)$. This paper models the arithmetic datapath verification problem as equivalence testing of polynomial functions from $Z_{2^{n_{1}}} \times Z_{2^{n_{2}}} \times \cdots \times Z_{2^{n_{d}}} \rightarrow Z_{2^{m}}$. We formulate the equivalence problem $f \equiv g$ into that of proving whether $f-g \equiv 0 \% 2^{m}$. Fundamental concepts and results from number, ring and ideal theory are subsequently employed to develop systematic, complete algorithmic procedures to solve the problem. We demonstrate application of the proposed theoretical concepts to high-level (behavioral/RTL) verification of bit-vector arithmetic within practical CAD settings. Using our approach, we verify a set of arithmetic datapaths at RTL where contemporary verification approaches prove to be infeasible.
\end{abstract}

Index Terms-Bit-Vector Arithmetic, Equivalence Checking, Finite Ring Algebra, Ideal theory

\section{INTRODUCTION}

RTL descriptions of integer datapaths that implement polynomial computations over bit-vectors are found in many practical designs; e.g., in Digital Signal Processing (DSP) for audio, video and multimedia applications [1]. The growing market for such applications requires sophisticated CAD support for design, analysis and verification. Such designs implement a sequence of ADD, MULT type of algebraic computations over bit-vectors for which contemporary verification frameworks do not possess the requisite modeling and manipulation capabilities. Polynomial algebra provides an ideal platform for modeling such arithmetic-intensive designs. However, the word-lengths of the bit-vector variables in the design are usually predetermined and fixed according to the desired precision. For correct modeling of such systems, the effect of these word-lengths need to be accounted for. For example, the largest (unsigned) integer value that a bit-vector of size $m$ can represent is $2^{m}-1$; implying that the bit-vector represents

Manuscript received ??? ??, 2006; revised ??? ??, 2006. This research is supported in part by grants from the National Science Foundation: (i) CCF0514966 \& (ii) CCF-515010. F. Enescu was also supported by a Georgia State University Research Initiation Grant.

N. Shekhar and P. Kalla are with the Department of Electrical and Computer Engineering, University of Utah, Salt Lake City, UT 84102 USA.

F. Enescu is with the Department of Mathematics and Statistics, Georgia State University, Atlanta, GA 30303 USA.

Personal use of this material is permitted. However, permission to reprint/republish this material for advertising or promotional purposes or for creating new collective works for resale or redistribution to servers or lists or to reuse any copyrighted component of this work in other works must be obtained from the IEEE. integer values reduced modulo $2^{m}\left(\% 2^{m}\right)$. This suggests that bit-vector arithmetic can be efficiently modeled as algebra over finite integer rings of residue classes $Z_{2^{m}}$ [2]. This requires the use of number, ring and ideal theory concepts for their manipulation.

This paper addresses the problem of equivalence verification of arithmetic datapath computations over bit-vectors where the operands have finite bit-widths. The problem is addressed at the algorithmic level, behavioral level or register-transfer level (RTL). Fig. 1 depicts the overall design flow for such applications and the corresponding verification problem as it appears in the context of our work.

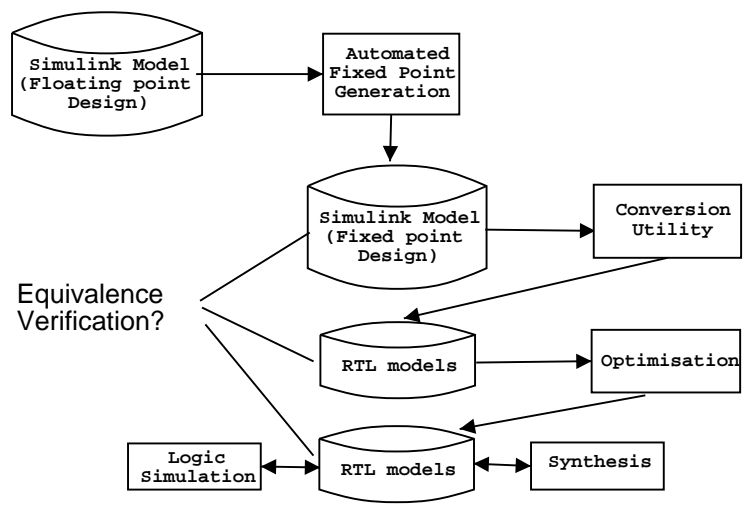

Fig. 1. The equivalence verification problem: Matlab to RTL design flow.

Initial algorithmic specifications (such as MATLAB models) of most signal processing applications involve data representation using floating-point formats. However, they are often implemented with fixed-point architectures in order to optimize the area, delay and power related costs of the implementations. Various automated tools exist for this translation [3]. Subsequently, the fixed-point model ("specification") can be translated into an RTL description ("implementation"); performed either manually, or by using automated conversion utilities, such as [4] [5]. The resulting RTL models can also be optimized using high-level synthesis/re-structuring operations (such as [6] [7]) [8]), leading to (bit-true) equivalent RTL descriptions. The verification problem is to prove that the fixedpoint model is computationally equivalent to the converted RTL model or to its optimized/transformed counterpart.

Let us consider specific problem instances that necessitate the use of finite ring algebra for equivalence testing purposes.

\section{A. Finite Word-length Bit-vector Arithmetic}

For polynomial datapath implementations, the design choice is often that of a uniform system word-length for the computations [9]. The datapath word-length is fixed to a constant 
(say, $m$ ) which is defined by the desired precision. In such cases, $m$-bit adders and multipliers produce an $m$-bit output; only the lower $m$-bits of the outputs are used and the higherorder bits are ignored. Usually, such computations require appropriate scaling of coefficients and/or signals such that 'overflow' can be avoided/ignored and standard fixed-point arithmetic can be implemented. When the datapath size $(m)$ over the entire design is kept constant, then fixed-size bitvector arithmetic manifests itself as polynomial algebra over the finite integer ring of residue classes $Z_{2^{m}}$; i.e. addition and multiplication is closed within the finite set of integers $\left\{0, \ldots, 2^{m}-1\right\}$. In such cases, symbolically distinct polynomials (those with different degrees and coefficients) can become computationally (bit-true) equivalent. The equivalence verification problem then reduces to that of proving the computational equivalence of polynomials: $f\left(x_{1}, x_{2}, \ldots, x_{d}\right) \% 2^{m} \equiv$ $g\left(x_{1}, x_{2}, \ldots, x_{d}\right) \% 2^{m}$, where $f, g$ are polynomials in $d$ variables $x_{1}, x_{2}, \ldots, x_{d}$ and $m=$ datapath size.

However, the fixed word-length paradigm is somewhat restrictive. Most designs usually contain operands with different word-lengths. For instance, a digital audio-video mixer, may perform polynomial arithmetic over a 20-bit audio and a 32bit video signal [1]. As a practical example, consider the computation performed by a digital image rejection/separation unit that takes as input two signals: a 12-bit vector $A[11: 0]$ and another 8 -bit vector $B[7: 0]$. These signals are outputs of a mixer wherein one signal emphasizes on the image signal and the other emphasizes on the desired signal. The design produces a 16-bit output $F$. The computation performed by the design is described in RTL as shown in Eqn. 1. Note that because of the specified bit-vector sizes, the computation can be equivalently implemented as another polynomial $G$, as shown in Eqn. 2.

input $A[11: 0], B[7: 0]$; output $F[15: 0], \quad G[15: 0]$;

$$
\begin{aligned}
F= & 16384 *\left(A^{4}+B^{4}\right)+64767 *\left(A^{2}-B^{2}\right)+A \\
& -B+57344 * A * B *(A-B) \\
G= & 24576 * A^{2} * B+15615 * A^{2}+8192 * A * B^{2}+ \\
& 32768 * A * B+A+17153 * B^{2}+65535 * B
\end{aligned}
$$

Such arithmetic datapaths with multiple word-length architectures can be analyzed as polynomial functions from $Z_{2^{n_{1}}} \times Z_{2^{n_{2}}} \times \cdots \times Z_{2^{n_{d}}} \rightarrow Z_{2^{m}}$ [10], where $n_{1}, n_{2}, \ldots, n_{d}$ are the bit-widths of input vectors and $m$ is the output width. So how do we prove the equivalence of such computations? Efficient algorithmic solutions to such problems is the subject of this paper.

\section{B. Problem Modeling}

We model the arithmetic computations over bit-vectors as follows. Let $x_{1}, x_{2}, \ldots, x_{d}$ denote the $d$-variables (bitvectors) in the design. Let $n_{1}, n_{2}, \ldots, n_{d}$ denote the size of the corresponding bit-vectors. Therefore, $x_{1} \in Z_{2^{n_{1}}}, x_{2} \in$ $Z_{2^{n_{2}}}, \ldots, x_{d} \in Z_{2^{n_{d}}}$. Here, $Z_{2^{n_{i}}}$ corresponds to the finite set of integers $\left\{0,1, \ldots, 2^{n_{i}}-1\right\}$. Let $m$ correspond to the size of the output bit-vector $f$; hence, $f \in Z_{2^{m}}$. Subsequently, we model the arithmetic datapath computation as a polynomial function (or polyfunction) over $Z_{2^{n_{1}}} \times Z_{2^{n_{2}}} \times \cdots \times Z_{2^{n_{d}}}$ to $Z_{2^{m}}$ [10]. Here $Z_{a} \times Z_{b}$ represents the Cartesian product of $Z_{a}$ and $Z_{b}$. In other words, the computation is modeled as a multivariate polynomial $F\left(x_{1}, x_{2}, \ldots, x_{d}\right) \% 2^{m}$, where each $x_{i} \in$ $Z_{2^{n_{i}}}$. The equivalence problem then corresponds to checking the congruence of two polynomials: $F \% 2^{m} \equiv G \% 2^{m}$. Note that modeling fixed-size datapaths can be treated as a special case of the above, where the bit-vectors sizes $n_{1}, n_{2}, \ldots, n_{d}$ are all equal to $m$ and the polynomial is computed over $Z_{2^{m}}\left[x_{1}, x_{2}, \ldots, x_{d}\right]$, denoted as $Z_{2^{m}}^{d}$.

\section{Solution Overview}

Solutions for $F\left(x_{1}, x_{2}, \ldots, x_{d}\right) \quad \% \quad 2^{m} \quad \equiv$ $G\left(x_{1}, x_{2}, \ldots, x_{d}\right) \% 2^{m}$ are available for fields $(R, Q, C)$, prime rings $Z_{p}$, Galois fields $\left(G F\left(p^{n}\right)\right)$, integral and Euclidean domains - collectively called the unique factorization domains (UFDs) [2]. In our context, the finite ring formed by the specific modulo value $2^{m}$ is a non-UFD, due to the presence of zero divisors (e.g., $4 \neq 2 \neq 0,4 \cdot 2=0 \% 8$ ), and correspondingly due to lack of multiplicative inverses. Unfortunately, this disallows the use of many efficient factorization-based techniques developed over UFDs.

The problem $F\left(x_{1}, \ldots, x_{d}\right) \% n=G\left(x_{1}, \ldots, x_{d}\right) \% n$ is decidable and is shown to be NP-Hard for $n \geq 2$ [11]. We transform our problem $F \% 2^{m} \equiv G \% 2^{m}$, into proving $(F-G) \% 2^{m} \equiv 0$; the well-known zero-equivalence problem [11]. For the example described in Eqns. $(1-2)$, we can compute $(F-G) \% 2^{16}$ :

$$
\begin{aligned}
F-G= & 16384\left(A^{4}+B^{4}\right)+32768 * A * B *(A+1)+ \\
& 49152 *\left(A^{2}+B^{2}\right)
\end{aligned}
$$

Note that $(F-G)$ is a polynomial with non-zero coefficients. However, $\forall A \in Z_{2^{12}}, B \in Z_{2^{8}},(F-G) \% 2^{16}=0$; i.e., $(F-$ $G)$ vanishes as a function from $Z_{2^{12}} \times Z_{2^{8}}$ to $Z_{2^{16}}$. Therefore, our problem reduces to that of testing whether or not $(F-G)$ is a vanishing polynomial $\% 2^{m}$.

Formulating the problem in this manner $(F-G \equiv 0)$ has its appeal because it belongs to a class of ideal membership testing problems. Moreover, properties of polynomial functions over finite rings have been well studied topics in number theory and commutative algebra [12] [13] [14] [10]. This paper analyzes and extends these results (particularly those of [13] and [10]) to derive systematic and efficient algorithmic procedures for equivalence checking, and demonstrates their application within a practical CAD-based verification framework.

\section{Scope of the Paper}

The approach presented in this paper has been applied to verify high-level descriptions of arithmetic datapaths, such as those in C and RTL (VERILOG/VHDL), some of which were automatically generated by MATLAB (Simulink and filter design toolboxes) [5]. Our technique is applicable to designs that implement unsigned and two's complement (overflow) arithmetic. In the DSP domain, rounding and saturation are 
also common modes of approximation. Modeling such architectures as polynomial functions over finite rings is significantly more involved and is not the subject of this paper. For the same reason, verification of (behavioral) RTL against its corresponding gate-level implementation (netlist) is also not dealt with in the paper. Even within this scope, we demonstrate that there exists a large class of applications where our approach can very efficiently solve the problem whereas contemporary verification techniques are infeasible.

\section{E. Paper Organization}

The paper is organized as follows: The next section reviews related work in VLSI-CAD, symbolic and polynomial algebra. Section III covers preliminary concepts regarding ring and ideal theory and describes how our problem relates to ideal membership testing. Section IV describes the mathematical aspects related to proving equivalence of univariate polynomials and provides a solution overview. These results are extended in Section V to provide an algorithm for multivariate computations with arbitrary input-output word-lengths. Section VI describes the experimental setup and compares our results with those of contemporary techniques. Finally, Section VII concludes the paper citing future research directions.

\section{REVIEW OF PREVIOUS WORK}

\section{A. VLSI-CAD}

A variety of canonical, directed acyclic graph (DAG) representations have been derived for Boolean functions: BDDs [15], *BMDs [16] and their various word-level extensions [17]. However, these are based on variants of bit-level (binary) decomposition principles. Hence, they lack the power of abstraction to model integer-level datapath computations. TEDs [18] have been proposed as canonical DAG representations for multi-variate polynomials. However, TEDs do not model modulo-arithmetic and hence cannot prove computational equivalence of polynomials over finite integer rings.

Verification techniques for bit-vector arithmetic such as arithmetic decision procedures, term-rewriting and others can be found in [19] [20]. Integer arithmetic [21] has also been used in constraint satisfaction for simulation-based validation. However, these techniques use modulo arithmetic concepts to solve linear congruences - a different application from proving polynomial equivalence in $Z_{2^{m}}$. There exist various DSP applications (such as FIR, IIR, Elliptical wave filters, etc.) whose RTL implementations are mostly linear and/or multi-linear expressions - these are somewhat easy to verify using theorem provers (HOL), congruence closure, dataindependence, symmetry and other abstractions [22] [23]. However, none of the above can efficiently solve the problem at hand.

\section{B. Ring/Field theory and Computer Algebra in VLSI-CAD}

Pradhan's work [24] represents the characteristic function of a circuit as polynomials over Galois Fields $\mathrm{GF}\left(2^{m}\right)$. Modulo arithmetic concepts have been employed in multiplier verification [25]. While these works find application at circuit-netlist level, they are not scalable enough to address polynomial bitvector computations.

Symbolic Algebra tools have been integrated with theorem provers [26] and also with high-level/RTL Synthesis tools [27] [6]. For example, [6] exploits Buchberger's seminal work on Grobner's bases [28] to decompose and synthesize a polynomial according to available components in the design library (using their Grobner's bases to decide). This work can exploit available algorithms from Maple [29] because their application is modeled over the field $R$ of real numbers or rationals $Q$. Unfortunately, porting these techniques to $Z_{2^{m}}$ is not straight-forward.

\section{Symbolic Algebra and Number Theory}

Ibarra et al. have extensively analyzed the decidability of equivalence and simplification of a variety of straight-line programs [11]. The zero-equivalence problem $f \% n \equiv 0$ is shown to be NP-hard for $n \geq 2$ [11]. Such polynomials that compute $0 \% n$ are called vanishing polynomials. For example, $F=4 x^{2}+4 x \equiv 0 \% 2^{3}, \forall x \in Z_{2^{3}}$; hence, $F$ is a vanishing polynomial in $Z_{2^{3}}$.

It is a well-known result that all vanishing polynomials over a finite ring are members of an ideal in that ring. Such vanishing ideals over finite sets of points have been studied over arbitrary fields [30]. This paper presents solutions to such problems in $Z_{2^{m}}$, within a CAD-based verification framework.

Over unique factorization domains (UFDs), the equivalence $f \equiv g$ can also be solved by factorizing the polynomials uniquely into their irreducible terms, and then comparing the coefficients of the ordered terms. Unfortunately, factorization is not unique in non-UFDs (such as in $Z_{2^{m}}$ ). Consider, for instance, $F(x)=x^{2}+6 x$ in $Z_{2^{3}}$. It can be factorized into two non-unique irreducibles: i) $(x)(x+6)$ and ii) $(x+2)(x+4)$, in $Z_{2^{3}}$. Therefore, unique factorization based techniques cannot be applied here. We have analyzed a large number of symbolic algebra packages [31] and, to the best of our knowledge, none of the available packages provide a 'ready-made' procedure that can solve the desired equivalence problem.

Researchers from number theory and commutative algebra have analyzed various properties of polynomial functions over finite rings. The next few sections review these results and extend them to derive systematic algorithmic procedures for our applications. First, some preliminary concepts.

\section{PRELIMINARIES}

This section briefly reviews basic commutative algebra concepts to put our polynomial equivalence problems in perspective. The material is mostly referred from [2].

Definition 3.1: A ring is a set $R$ with two binary operations $'{ }^{\prime}{ }^{\text {and }}{ }^{\prime} . '$ (addition and multiplication) satisfying additive and multiplicative associativity, additive commutativity, left and right distributivity, and existence of additive identity and inverse. A commutative ring also satisfies multiplicative commutativity.

The set $Z_{n}=\{0,1, \ldots, n-1\}$, where $n \in N$, also forms a commutative ring with unity. It is called the residue class ring, 
where addition and multiplication are defined modulo $n(\% n)$ according to the rules below. For our application, $n=2^{m}$.

$$
\begin{aligned}
(a+b) \% n & =(a \% n+b \% n) \% n \\
(a \cdot b) \% n & =(a \% n \cdot b \% n) \% n \\
(-a) \% n & =(n-a \% n) \% n
\end{aligned}
$$

Definition 3.2: Integers $x, y$ are called congruent modulo $n(x \equiv y \% n)$ if $n$ is a divisor of their difference: $n \mid(x-y)$.

Definition 3.3: A zero divisor is a non-zero element $x$ of a ring $R$, for which $x \cdot y \equiv 0$, where $y$ is some other non-zero element of $R$ and the multiplication $x \cdot y$ is defined according to Eqn. (5).

Definition 3.4: A fi eld $F$ is a commutative ring with unity, where every element in $F$, except 0 , has a multiplicative inverse, i.e. $\forall a \in F-\{0\}, \exists \hat{a} \in F$ such that $a \cdot \hat{a}=1$.

The system $Z_{n}$ forms a field if and only if $n$ is prime. Hence, $Z_{2^{m}}$ (for $m>1$ ) is not a field as not every element in $Z_{2^{m}}$ has an inverse. Lack of inverses in $Z_{2^{m}}$ makes RTL verification complicated since Euclidean algorithms for division and factorization are no longer applicable.

Definition 3.5: Let $R$ be a ring. A polynomial over $R$ in the indeterminate $x$ is an expression of the form:

$$
a_{0}+a_{1} x+a_{2} x^{2}+\cdots+a_{k} x^{k}=\sum_{k} a_{i} x^{i}
$$

$\forall a_{i} \in R$. Elements $a_{i}$ are coefficients, $k$ is the degree. The element $a_{k}$ is called the leading coeffi cient; when $a_{k}=1$, the polynomial is monic.

The system consisting of the set of all polynomials in $x$ over the ring $R$, with addition and multiplication defined accordingly, also forms a ring, called the ring of polynomials $R[x]$. Similarly, $R\left[x_{1}, \ldots x_{d}\right]$ denotes a ring of multi-variate polynomials in $d$ variables. Note that, when $R=Z_{2^{m}}$, the corresponding polynomials are evaluated $\% 2^{m}$.

Functions over finite rings that can be represented by polynomials are generally termed as polynomial functions (or polyfunctions). Singmaster [13] studied various properties of polynomial functions of the type $f: Z_{n} \rightarrow Z_{n}$. Subsequently, Chen extended the study to analyze polyfunctions of the type $f: Z_{n} \rightarrow Z_{m}$ [14] and further to those of the type $f: Z_{n_{1}} \times Z_{n_{2}} \times \cdots \times Z_{n_{d}} \rightarrow Z_{m}$ [10]. The following definition of such a polynomial function is taken from [10], and modified, for our application, to rings modulo an integer power of 2.

Definition 3.6: A function $f$ from $Z_{2^{n_{1}}} \times Z_{2^{n_{2}}} \times \ldots \times$ $Z_{2^{n} d} \rightarrow Z_{2^{m}}$ is said to be a polynomial function (or polyfunction) if it is represented by a polynomial $F \in$ $Z\left[x_{1}, x_{2}, \ldots, x_{d}\right]$; i.e. $f\left(x_{1}, x_{2}, \ldots, x_{d}\right) \equiv F\left(x_{1}, x_{2}, \ldots, x_{d}\right)$ for all $x_{i} \in Z_{2^{n_{i}}}, i=1,2, \ldots, d$ and $\equiv$ denotes congruence $\left(\bmod 2^{m}\right)$.

Example 3.1: Let $f: Z_{2^{1}} \times Z_{2^{2}} \rightarrow Z_{2^{3}}$ be a polyfunction in variables $x, y$, defined as: $f(0,0)=1, f(0,1)=3$, $f(0,2)=5, f(0,3)=7, f(1,0)=1, f(1,1)=4, f(1,2)=$ $1, f(1,3)=0$. Then, $f$ is a polyfunction representable by $F=1+2 y+x y^{2}$, since $f(x, y) \equiv F(x, y) \% 2^{3}$ for $x=0,1$ and $y=0,1,2,3$.
It is possible for a polynomial with non-zero coefficients to vanish on such mappings; in which case the polynomials are called vanishing polynomials and their functions correspond to nil polyfunctions. For example, the polynomial $4 x^{2}+4 x \equiv$ $0 \% 8, \forall x \in Z_{8}$; hence $4 x^{2}+4 x$ is a vanishing polynomial in $Z_{8}[x]$.

\section{A. Ideals and Ideal Membership Testing}

It is important to note that the set of all vanishing polynomials in a given ring forms an ideal in that ring. Thus, to prove that $F-G \equiv 0 \% 2^{m}$, we need to determine if $(F-G)$ is a member of the vanishing ideal in $Z_{2^{m}}$.

Definition 3.7: Let $I$ be a subset of the ring $R$. Then $I$ is called an ideal of $R$ if

- $0 \in I$

- $I$ is closed under addition; $x, y \in I \Rightarrow x+y \in I$

- If $x \in R$ and $y \in I$, then $x \cdot y \in I$ as well as $y \cdot x \in I$.

Definition 3.8: Let $i_{1}, i_{2}, \ldots, i_{n}$ be the given elements of the commutative ring $R$. Let $I$ be an ideal of $R$. If:

$$
I=\left\{x_{1} i_{1}+x_{2} i_{2}+\ldots+x_{n} i_{n}: x_{1}, \ldots, x_{n} \in R\right\}
$$

then, $i_{1}, \ldots, i_{n}$ are called the generators of the ideal $I$ and we denote this as $I=\left(i_{1}, i_{2}, \ldots, i_{n}\right)$.

With regard to our application, it is required to analyze the generators of the ideal of all vanishing polynomials in $Z_{2^{m}}$. The works of [12] and [13] have shown that this ideal can be finitely generated over any arbitrary finite integer ring $\left(Z_{n}\right)$. Therefore, we begin with a discussion of the vanishing ideal in $Z_{2^{m}}[x]$ and subsequently provide a unique representative expression for all members of this ideal. Sec. IV outlines an efficient algorithm to determine if any given polynomial vanishes by checking if it corresponds to this unique form. These concepts are extended to polyfunctions in $d$ variables from $Z_{2^{n_{1}}} \times Z_{2^{n_{2}}} \times \ldots \times Z_{2^{n_{d}}}$ to $Z_{2^{m}}$, in Section V. Examples are used to demonstrate relevant results; their corresponding proofs can be found in [13] and [10] and are therefore not reproduced here.

\section{On UniVARIATE VANishing POLYNOMials}

According to a fundamental result in number theory, for any $n \in N, n$ ! divides the product of $n$ consecutive numbers. For example, 4 ! divides $4 \times 3 \times 2 \times 1$. This is also true of any $n$ consecutive numbers: 4 ! also divides $99 \times 100 \times 101 \times 102$. Consequently, it is possible to find the least $k \in N$ such that $n \mid k$ !. We denote this value $k$ as $\mathbf{S F}(\mathbf{n})$ [32], i.e. $k=S F(n)^{1}$. In the ring of interest, $Z_{2^{m}}$, let $S F\left(2^{m}\right)$ be equal to the least integer $k$, such that $2^{m} \mid k$ !. As an example, $S F\left(2^{3}\right)=4$ as 8 divides 4 ! $(=4 \times 3 \times 2 \times 1=8 \times 3)$. Note that 8 does not divide 3 !, and hence the least $k$ in question $=4$.

The significance of the above concept can be explained as follows. Consider the ring $Z_{2^{3}}$. The least value $k$ such that

\footnotetext{
${ }^{1}$ This is a well-studied function in number theory. It was initially studied by Kempner [33] and was recently re-visited upon by Smarandache [32]. In recent literature it is often referred to as the Smarandache function and hence we refer to it as $S F(n)$.
} 
$8 \mid k !$ is $k=4$. Therefore, any integer that can be factored into a product of (at least) $k=4$ consecutive numbers will vanish in $Z_{2^{3}}$. This property can be utilized to treat the equivalence problem as a divisibility issue in $Z_{2^{m}}$, i.e. $f(x)-g(x) \equiv 0 \Rightarrow$ $2^{m} \mid(f(x)-g(x))$. In $Z_{2^{3}}$, let $8 \mid(f(x)-g(x))$. But, $8 \mid 4$ ! too. Therefore, if $(f-g)$, evaluated at $x$, can be represented as the product of 4 consecutive numbers (depending on $x$ ), then $(f-g)$ would vanish in $Z_{2^{3}}$. So, what is a natural example of a polynomial with this property? The answer is: $(x)(x-$ 1) $(x-2)(x-3)$.

In this regard, Chen [14] proposed a set of monic polynomials, $Y_{k}(x)$, where each $Y_{i}(x)$ represents (in polynomial form) a product of $i$ consecutive numbers in $x$. More formally, we have the following definition and its corresponding result:

Definition 4.1: Falling factorials of degree $k$ are defined according to:

- $Y_{0}(x)=1$

- $Y_{1}(x)=x$

- $Y_{2}(x)=x(x-1)$

\section{- $Y_{k}(x)=(x-k+1) \cdot Y_{k-1}(x)$}

Lemma 4.1: Any expression in $Z_{2^{m}}[x]$ that can be factored into at least $Y_{k}(x)$ (where $k=S F\left(2^{m}\right)$ ), will be divisible by $2^{m}$ and vanish.

Example 4.1: Consider the polynomial $F(x)$ corresponding to the function $f: Z_{2^{8}}[x] \rightarrow Z_{2^{8}}$ :

$$
\begin{aligned}
F(x)= & x^{10}+211 x^{9}+102 x^{8}+22 x^{7}+41 x^{6}+ \\
& 243 x^{5}+224 x^{4}+36 x^{3}+16 x^{2}+128 x
\end{aligned}
$$

In $Z_{2^{8}}[x], S F\left(2^{8}\right)=10$. Therefore, if for all $x, F(x)$ can be factored into a product of 10 consecutive numbers, (as in the case of $Y_{10}(x)$ ), then $F(x)$ is a vanishing polynomial in $Z_{2^{8}}[x]$. Indeed, $F(x) \equiv Y_{10}(x) \% 2^{8}$; hence $F(x) \% 2^{8} \equiv 0$.

When a polynomial cannot be factored into such $Y_{k}$ expressions, can it still vanish? For example, the quadratic vanishing polynomial $4 x^{2}-4 x$ in $Z_{2^{3}}$, written as $4(x)(x-1)$, cannot be factorized as $Y_{4}(x)=x(x-1)(x-2)(x-3)$. However, the missing factors, $(x-2)(x-3)$ in this case, are compensated for by the multiplicative constant 4 ; therefore, $4 x^{2}-4 x \equiv 0 \% 2^{3}$. Singmaster [13] identified the constraint on such multiplicative constants such that the polynomial in question would vanish. We state the following result.

Lemma 4.2: The expression $c_{k} \cdot Y_{k}(x) \equiv 0$ in $Z_{2^{m}}[x]$ if and only if $\frac{2^{m}}{\left(k !, 2^{m}\right)} \mid c_{k}$;

where,

- $c_{k}$ is an arbitrary integer in $Z$,

- $Y_{k}(x)$ is as defined above,

- $\left(k !, 2^{m}\right)$ is the greatest common divisor (GCD) of $k$ ! and $2^{m}$, and

- $k$ is the degree of the expression $c_{k} \cdot Y_{k}(x)$.

Example 4.2: Let us explain the above concept with the help of the previous example. Let $F(x)=4 x^{2}-4 x$ over $Z_{2^{3}}[x]$. Note that $F(x)=4(x)(x-1)=4 \cdot Y_{2}(x)$. Therefore, in this case $k=2$ and $c_{2}=4$; and $\frac{2^{3}}{\left(2 !, 2^{3}\right)}(=4)$ divides $c_{2}(=4)$.
Because the above condition is satisfied, $F(x) \% 2^{3} \equiv 0$. Note if $c_{2}$ were replaced by 3 , then $F(x)=3(x)(x-1)$ would not be a vanishing polynomial as $\frac{2^{3}}{\left(2 !, 2^{3}\right)}$ would not divide 3 .

The above concepts lead to Singmaster's theorem [13] that identifies the necessary and sufficient conditions for a polynomial to vanish over any finite integer ring. We re-state the result for $Z_{2^{m}}$.

Theorem 1: Let $F$ be a polynomial in $Z_{2^{m}}[x]$. Then $F$ vanishes $\% 2^{m}$ if and only if:

$$
F \equiv F_{n} \cdot Y_{n}+\sum_{k=0}^{n-1} a_{k} \cdot b_{k} \cdot Y_{k}
$$

where,

- $n=S F\left(2^{m}\right)$, i.e. the least $n$ such that $2^{m} \mid n$ !,

- $F_{n}$ is an arbitrary polynomial,

- $a_{k}$ is an arbitrary integer,

- $b_{k}=\frac{2^{m}}{\left(k !, 2^{m}\right)}$, as described in Lemma 4.2.

Note that the expression $F_{n} \cdot Y_{n}+\sum_{k=0}^{n-1} a_{k} \cdot b_{k} \cdot Y_{k}$ in Theorem 1 above completely describes the ideal of all vanishing polynomials over $Z_{2^{m}}[x]$. Thus, if any polynomial $(F-G)$ can be described as above, then $F-G \equiv 0 \% 2^{m}$, implying that $F \% 2^{m} \equiv G \% 2^{m}$.

Example 4.3: Consider $F=4 x^{2}-4 x=4(x)(x-1)$ in $Z_{2^{3}}[x]$. Again, $n=S F\left(2^{3}\right)=4$. However, $F$ cannot be factored into $Y_{4}(x)$, therefore $F_{4}=0$.

Now consider $k=n-1=4-1=3$. Since $4 x^{2}-4 x$ (quadratic) cannot be factored by $Y_{3}(x)$ (cubic), $a_{3}=0$. However, $F$ can be factored according to $Y_{2}$, leading to $a_{2}=1$ and $b_{2}=4$. Therefore, $F$ vanishes in $Z_{2^{3}}$.

\section{A. Algorithm}

From the above results, a systematic, complete, algorithmic procedure can be derived to identify whether or not a given polynomial corresponds to the unique form of Eqn. (9) and consequently, vanishes over rings of the form $Z_{2^{m}}$ ( $m$ is the datapath size). The algorithm is given in Fig. 3. The inputs are the given uni-variate polynomials, $F$ and $G$, in variable $x$ with a uniform bit-width of $m$ bits. The main procedure is outlined below:

1) Compute $n=S F\left(2^{m}\right)$; the computational procedure is outlined in Fig. 2.

2) Iteratively divide the polynomial (poly) by the $Y_{k}(x)$ expressions, for $k=n$ down to 0 .

3) If $k=n$ and $Y_{n} \mid$ poly, then poly $=F_{n} \cdot Y_{n}$, a vanishing polynomial.

4) If the degree of poly is less than $k$ in any iteration, then $F_{n}=0$ and/or $a_{k}=0$. Continue the division procedure with $Y_{k-1}$.

5) Otherwise check for the constraints on $a_{k}, b_{k}$. If the constraints are not satisfied, then it is not a vanishing polynomial. If the constraints are satisfied, then the procedure iterates over the remaining terms.

6) The procedure converges with the correct answer on whether or not poly $\equiv 0$ in $Z_{2^{m}}[x]$. 


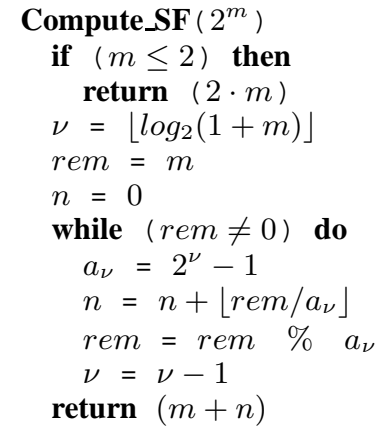

Fig. 2. $\mathrm{SF}\left(2^{m}\right)$ computation.

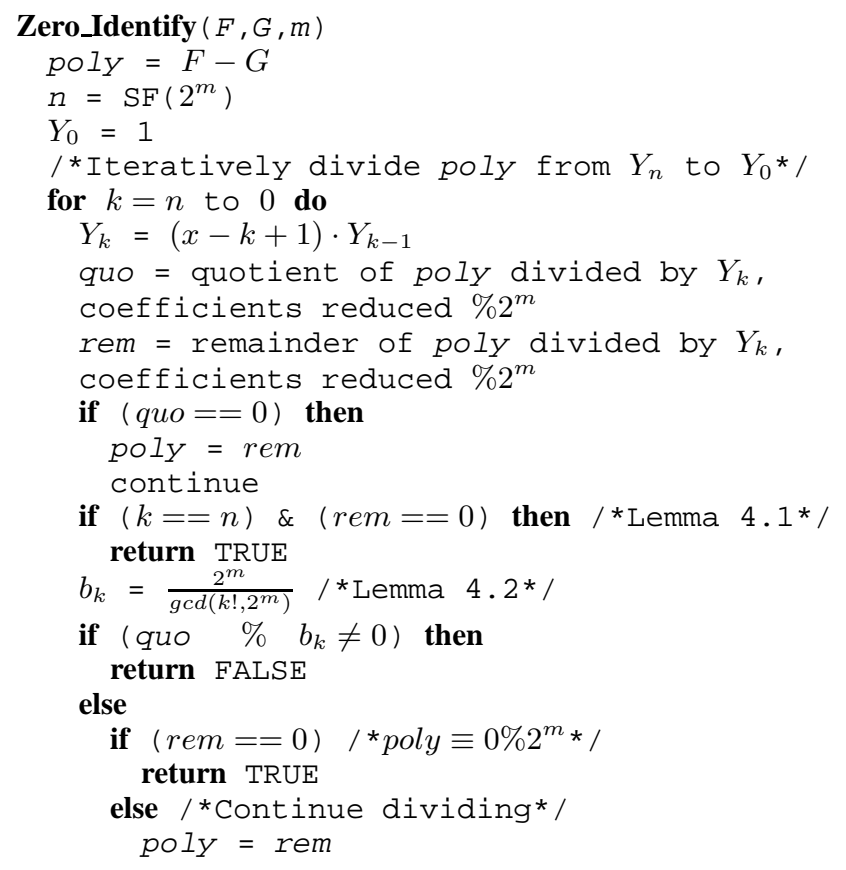

Fig. 3. Zero_Identify - Determine whether the algebraic difference of polynomials $F$ and $G$ vanishes $\% 2^{m}$.

Complexity: In the worst case, $(n+1)$ divisions by $Y_{k}$ expressions are performed on poly. Moreover, in each iteration, the $Y_{k}$ computation requires $O(n)$ multiplications. The worstcase complexity of the algorithm is, therefore, $O\left(n^{2}\right)$, where $n=S F\left(2^{m}\right)$.

\section{Equivalence of Multi-VARiate Polynomials}

We now proceed to extend the results of the previous section to polynomials in $d$ variables. In Section I, we had shown how the multiple-word-length bit-vector computation can be modeled as a polynomial function from $Z_{2^{n_{1}}} \times Z_{2^{n_{2}}} \times \cdots \times Z_{2^{n} d}$ to $Z_{2^{m}}$. Moreover, we had noted that a fixed-size datapath with multiple variables is a special case of the above, where $n_{1}=\cdots=n_{d}=m$. Furthermore, we had sought to exploit the concept of $S F\left(2^{m}\right)$ and that of the falling factorials $Y_{k}(x)$, to detect vanishing polynomials over $Z_{2^{m}}[x]$. We will now extend these concepts to address multi-variate polynomials.

Definition 5.1: Let $\mathbf{k}=\left\langle k_{1}, k_{2}, \ldots, k_{d}\right\rangle \in\left(Z^{+}\right)^{d}$, where
$Z^{+}$denotes the set of non-negative integers. We define,

$$
\mathbf{Y}_{\mathbf{k}}=\prod_{i=1}^{d} Y_{k_{i}}\left(x_{i}\right)=Y_{k_{1}}\left(x_{1}\right) \cdot Y_{k_{2}}\left(x_{2}\right) \cdots Y_{k_{d}}\left(x_{d}\right)
$$

where $Y_{k_{i}}\left(x_{i}\right)$ is the falling factorial of degree $k_{i}$ in variable $x_{i}$, as defined in Definition 4.1.

Lemma 4.1 is now applicable over $Z_{2^{m}}\left[x_{1}, \ldots, x_{d}\right]$ as well. If a multivariate polynomial in $Z_{2^{m}}\left[x_{1}, \ldots, x_{d}\right]$ can be factorized into a product of $S F\left(2^{m}\right)$ consecutive numbers in at least one of the variables $x_{i}$, then it vanishes $\% 2^{m}$. The following example illustrates this idea.

Example 5.1: Consider the polynomial $F\left(x_{1}, x_{2}\right)=$ $x_{1}^{4} x_{2}+2 x_{1}^{3} x_{2}+3 x_{1}^{2} x_{2}+2 x_{1} x_{2}$ over $Z_{2^{2}}\left[x_{1}, x_{2}\right]$. Here, $S F\left(2^{2}\right)=4$. Note that we can write $F=Y_{\langle 4,1\rangle}\left(x_{1}, x_{2}\right) \% 4=$ $Y_{4}\left(x_{1}\right) \cdot Y_{1}\left(x_{2}\right) \% 4$. Since $F$ can be represented as a product of 4 consecutive numbers in $x_{1}, 2^{2} \mid F$ and $F \equiv 0 \% 4$.

In the above example, both the input variables $x_{1}, x_{2}$, as well as the output $F$ are in $Z_{2^{2}}$. We wish to generalize these results to analyze polynomial functions over $Z_{2^{n_{1}}} \times Z_{2^{n_{2}}} \times$ $\cdots \times Z_{2^{n} d_{d}} \rightarrow Z_{2^{m}}$. For this purpose, another quantity, $\mu_{i}$, is defined as follows [10]:

$$
\mu_{i}=\min \left\{2^{n_{i}}, S F\left(2^{m}\right)\right\} ; i=1,2, \ldots, d
$$

We now present the following results from [10]:

Lemma 5.1: Let $\mathbf{k}=\left\langle k_{1}, k_{2}, \ldots, k_{d}\right\rangle \in\left(Z^{+}\right)^{d}$, where $Z^{+}$ denotes the set of non-negative integers. Then, $\mathbf{Y}_{\mathbf{k}} \equiv 0$ if and only if $k_{i} \geq \mu_{i}$, for some $i$.

Example 5.2: Let $F=x_{1}^{2} x_{2}-x_{1} x_{2}$ be a polynomial corresponding to the polyfunction $f: Z_{2^{1}} \times Z_{2^{2}} \rightarrow Z_{2^{3}}$. We show that $F$ is a vanishing polynomial as $F$ can be written according to:

$$
\begin{aligned}
x_{1}^{2} x_{2}-x_{1} x_{2} & \equiv x_{1}\left(x_{1}-1\right) x_{2} \\
& \equiv Y_{\langle 2,1\rangle}\left(x_{1}, x_{2}\right) \\
& \equiv 0
\end{aligned}
$$

Here, $S F\left(2^{3}\right)=4, k_{1}=2$ and $k_{2}=1$. Note that $\mu_{1}=$ $\min \left\{2^{1}, 4\right\}=2=k_{1}$ and $\mu_{2}=\min \left\{2^{2}, 4\right\}=4>k_{2}$. Since $k_{1} \geq \mu_{1}$, the condition in Lemma 5.1 is satisfied, and hence $F \equiv 0$.

Lemma 5.2: The expression $c_{\mathbf{k}} \cdot \mathbf{Y}_{\mathbf{k}} \equiv 0$ if and only if $\frac{2^{m}}{\left(2^{m}, \prod_{i=1}^{d} k_{i} !\right)} \mid c_{\mathbf{k}}$; where:

- $c_{\mathbf{k}} \in Z$;

- $\mathbf{k}=\left\langle k_{1}, k_{2}, \ldots, k_{d}\right\rangle \in\left(Z^{+}\right)^{d}$ such that $k_{i}<\mu_{i}, \forall i=$ $1, \ldots, d ;$ and

- $\left(2^{m}, \prod_{i=1}^{d} k_{i}\right.$ !) is the greatest common divisor (GCD) of $2^{m}$ and $\prod_{i=1}^{d} k_{i}$ !.

Example 5.3: Consider the polynomial $F=4 x_{1} x_{2}^{2}+4 x_{1} x_{2}$ corresponding to $f\left(x_{1}, x_{2}\right): Z_{2^{1}} \times Z_{2^{2}} \rightarrow Z_{2^{3}}$. We can use Lemma 5.2 to prove that $f$ is a nil polyfunction. Here, $2^{n_{1}}=$ $2,2^{n_{2}}=4$ and $2^{m}=8$. Also, $\operatorname{SF}\left(2^{m}=8\right)=4 ; \mu_{1}=$ $\min \{2,4\}=2, \mu_{2}=\min \{4,4\}=4$. 


$$
\begin{aligned}
F & \equiv 4 x_{1} x_{2}^{2}+4 x_{1} x_{2} \\
& \equiv 4 \cdot x_{1} \cdot x_{2} \cdot\left(x_{2}-1\right) \\
& \equiv c_{\langle 1,2\rangle} \cdot Y_{\langle 1,2\rangle}\left(x_{1}, x_{2}\right) \\
& \equiv 0
\end{aligned}
$$

because $\frac{2^{m}}{\left(2^{m}, \prod_{i=1}^{d} k_{i} !\right)}=\frac{8}{(8,1 ! \cdot 2 !)}=4$ which divides $c_{\langle 1,2\rangle}=4$. Also note that here $k_{1}<\mu_{1}$ and $k_{2}<\mu_{2}$.

Chen extended the above results to derive necessary and sufficient conditions for a polynomial to vanish as a function from $Z_{2^{n_{1}}} \times Z_{2^{n_{2}}} \times \cdots Z_{2^{n_{d}}}$ to $Z_{2^{m}}$. We state the following theorem [10]:

Theorem 2: Let $F$ be a polynomial representation for the function $f$ from $Z_{2^{n_{1}}} \times Z_{2^{n_{2}}} \times \cdots \times Z_{2^{n_{d}}}$ to $Z_{2^{m}}$. Then $F$ is a vanishing polynomial $(F \equiv 0)$ if and only if it can be represented as:

$$
F=\sum_{i=1}^{d} Q_{i} Y_{\mu(\mathbf{i})}+\Sigma_{\mathbf{k}} a_{\mathbf{k}} b_{\mathbf{k}} \mathbf{Y}_{\mathbf{k}}
$$

where:

- $\mu(\mathbf{i})=\left\langle 0, \ldots, \mu_{i}, \ldots, 0\right\rangle$ is a $d$-tuple, where $\mu_{i}$ is in position $i$ and $\mu_{i}$ is defined according to Eqn.(11);

- $Q_{i} \in Z\left[x_{1}, \ldots, x_{d}\right]$ are arbitrary polynomials, possibly zero;

- $Y_{\mu(\mathbf{i})}$ is the falling factorial of degree $\mu_{i}$ in variable $x_{i}$;

- $\mathbf{k}=\left\langle k_{1}, \ldots, k_{d}\right\rangle$ for each $k_{i}=0,1, \ldots, \mu_{i}-1$;

- $\mathbf{Y}_{\mathbf{k}}$ is as defined in Eqn. (10);

- $a_{\mathbf{k}} \in Z$ is an arbitrary integer and

- $b_{\mathbf{k}}=\frac{2^{m}}{\left(2^{m}, \prod_{i=1}^{d} k_{i} !\right)}$.

Proof: While a detailed proof of the above theorem is provided in [10], the theorem does follow from Lemma 5.1 (for each of the $d$ computations $Q_{i} Y_{\mu(\mathbf{i})}$ ) and from Lemma 5.2 (for the computation $\Sigma_{\mathbf{k}} a_{\mathbf{k}} b_{\mathbf{k}} \mathbf{Y}_{\mathbf{k}}$ ).

The following example illustrates the above concept.

Example 5.4: Consider a polynomial $F=x_{1}^{2}+7 x_{1}+$ $4 x_{1} x_{2}^{2}+4 x_{1} x_{2}$ for $f: Z_{2} \times Z_{2^{2}} \rightarrow Z_{2^{3}}$. Here, $d=2, \mu_{1}=$ $\min \{2, S F(8)\}=2 ; \mu_{2}=\min \left\{2^{2}, S F(8)\right\}=4$. Therefore, $\mu(\mathbf{1})=\left\langle\mu_{1}, 0\right\rangle=\langle 2,0\rangle$, and hence $Y_{\mu(\mathbf{1})}=Y_{\langle 2,0\rangle}\left(x_{1}, x_{2}\right)$. Similarly, $\mu(\mathbf{2})=\left\langle 0, \mu_{2}\right\rangle=\langle 0,4\rangle$. Now, $F$ can be written as follows:

$$
\begin{aligned}
F & \equiv x_{1}\left(x_{1}-1\right)+4 \cdot x_{1} \cdot x_{2} \cdot\left(x_{2}-1\right) \\
& \equiv Y_{\langle 2,0\rangle}\left(x_{1}, x_{2}\right)+a_{\langle 1,2\rangle} b_{\langle 1,2\rangle} Y_{\langle 1,2\rangle}\left(x_{1}, x_{2}\right) \\
& \equiv 0
\end{aligned}
$$

Here, $Q_{1}=1, a_{\langle 1,2\rangle}=1$ and $b_{\langle 1,2\rangle}=8 /(8,1 ! \cdot 2 !)=4$. Note that $Q_{2}$ and all remaining $a_{\mathbf{k}}$ terms are equal to 0 . Hence, $F$ can be written in the form given by Theorem 2 , and is thus a vanishing polynomial.

Again, Eqn. (12) completely describes the ideal of all vanishing polynomials in $d$ variables over $Z_{2^{n_{1}}} \times Z_{2^{n_{2}}} \times \cdots Z_{2^{n_{d}}}$ to $Z_{2^{m}}$. We now describe an algorithm which determines the equivalence of any two given polynomials $F$ and $G$ by determining if $F-G$ can be reduced to this form, implying that $F-G \equiv 0 \% 2^{m}$.

\section{A. Algorithm}

The algorithm takes as input the two polynomials $F$ and $G$ in variables $x_{1}, \ldots, x_{d}$ with corresponding bit-widths $n_{1}, \ldots, n_{d}$. The output is true if $F \equiv G$. The algorithm is given in Fig. 4, the main procedure of which is outlined below:

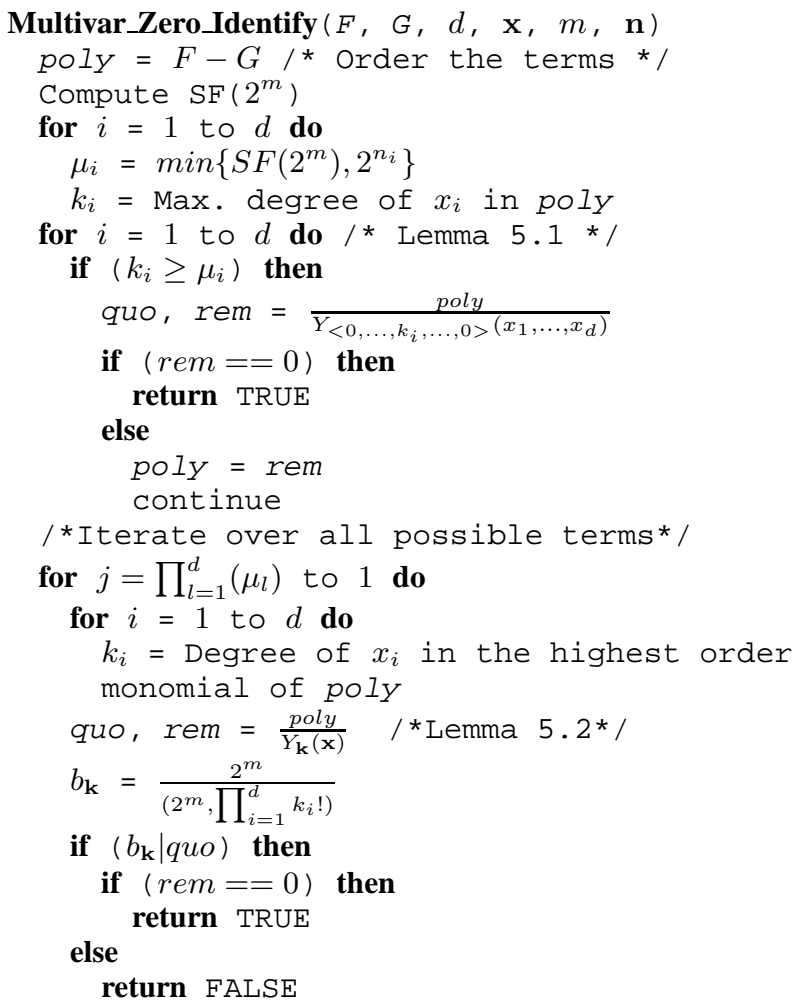

Fig. 4. Algorithm Multivar_Zero_Identify: Determine whether the algebraic difference of multivariate polynomials $F$ and $G$ vanishes.

1) Find the difference of the two polynomials, poly. This is the expression which should vanish to prove equivalence.

2) Order the monomial terms of poly in a descending lexicographic order on the variables. Monomials are compared by their degree in the first variable, with ties broken using the degrees in the second variable, third variable and so on.

3) Compute $S F\left(2^{m}\right)$. Use this to obtain the $\mu_{i}$ values.

4) Divide the polynomial by the falling factorial expressions $Y_{\mu(\mathbf{i})}$ in each of the $d$ variables. In any iteration, if the remainder is zero, it means that $F=\sum_{i=1}^{d} Q_{i} Y_{\mu(\mathbf{i})}+$ 0 ; hence return true. It is a vanishing polynomial; or $F \equiv G$.

5) If the above process results in a non-zero remainder, we have to see whether the remainder can be expressed as $\Sigma_{\mathbf{k}} a_{\mathbf{k}} b_{\mathbf{k}} \mathbf{Y}_{\mathbf{k}}$. For this purpose, use the subsequent remainder as the new poly. Update the degrees $\left(k_{i}\right)$ and continue dividing from $Y_{\mu-1}$ to $Y_{0}$ for each variable.

6) After each division, check for the following conditions:

- If the quotient can be written as $a_{\mathbf{k}} \cdot b_{\mathbf{k}}$ (where $b_{\mathbf{k}}$ is defined according to Lemma 5.2), and the remainder is zero, return true. It is a vanishing polynomial. 
- If the quotient can be written as $a_{\mathbf{k}} \cdot b_{\mathbf{k}}$, and the remainder is non-zero, continue to the next iteration.

- If the quotient cannot be written as $a_{\mathbf{k}} \cdot b_{\mathbf{k}}$, return false. poly $\neq 0 \Rightarrow F \neq G$.

In the worst case, $\left(\mu_{i}\right)$ divisions by the $Y_{\mathbf{k}}$ expressions are performed on poly for every $i=1, \ldots, d$.

\section{EXPERIMENTAL SETUP AND RESULTS}

We have implemented the proposed algorithms in Perl with calls to MAPLE 7 [29] for all the algebraic manipulations. Using our algorithms, we have been able to perform verification runs over a number of designs collected from a variety of benchmark suites. The results are presented in Table I.

The first set of examples are datapaths with a single input bit-vector variable, which are modeled as univariate polynomials. The anti-aliasing function is from [6]. The second example is a polynomial expression from [34]. The other univariate examples are implementations of elementary function computations. The first benchmark in the set of multivariate datapath instances represents an image rejection computation (IRR), as described in Sec. I. The phase-shift keying (PSK) function is from [6] and is used in digital communication. The polynomial filters are Volterra models of polynomial signal processing applications taken from [1]. MIBENCH is a $9^{t h}$ degree polynomial from a set of automotive applications in [35]. Horner polynomials are from [34]. Polynomial computations commonly used in DSP are often implemented in Horner's form using multiply-add-accumulate (MAC) units. In [6], it was shown how computations by these MAC units can be extracted as polynomials in Horner's form. The vanishing polynomial examples, for both the univariate and multivariate cases, were specifically created to validate our algorithms.

Some of these designs are available as RTL code. The others were available as high-level specifications in MATLAB or $\mathrm{C}$ code. RTL code for these reference designs was automatically generated using the MATLAB Simulink and Filter Design toolboxes (particularly for the digital filter designs). Once the reference RTL descriptions were obtained, they were further optimized using techniques from [6] and [8]. In [6], application of high-level restructuring and symbolic algebra-based transformations was presented for high-level synthesis. These include factorization and expansion, treeheight reduction, etc. The recent work of [8] has derived a sequence of polynomial algebra based transformations to reduce the area-cost of the implementation. This is achieved by modulating and segmenting the coefficients and subsequently removing algebraic redundancy (vanishing polynomials). In essence, the technique of [8] attempts to search for a sparser implementation of a given polynomial that occupies lesser area. These transformations were applied to the original RTL description to obtain functionally equivalent implementations. The optimized RTL was then verified for equivalence against the original one.

For equivalence testing, both RTL descriptions were given to the high-level synthesis tool GAUT [36] and their corresponding data-flow graphs (DFGs) were extracted. Traversing the DFGs from the inputs to the outputs, the polynomial representations were constructed and the datapath sizes were noted. The algorithms were invoked to find the difference between the two polynomials (both univariate and multivariate) and subsequently verified that it computes zero, to prove equivalency. We were able to solve all problems in $<5$ seconds.

We have also performed equivalence checking of the given RTL designs using BDDs, BMDs and SAT based approaches.

BDD and SAT: Since gate-level descriptions are required by both BDDs and SAT, we synthesized our designs using a commercially available logic synthesis tool. BDDs were used to verify the resulting netlists using the VIS [37] package. It was found that BDDs could solve the problem for some of the smaller benchmarks (especially for univariate polynomials) due to the simplification achieved by propagating the corresponding coefficients (constants). However, they failed for the rest of the designs.

From the gate-level netlists corresponding to the two designs, we generated miter circuits and converted them to CNF format. ZChaff [38] was used to prove equivalence via unsatisfiability testing. For all the designs, ZChaff could not solve the problem within the time-limit of 1000 s.

*BMD: *BMDs have been shown to be effective for multiplier verification, as they have linear size complexity for multipliers. However, for higher degree $(k)$ polynomials, their size increases $O\left(n^{k}\right)$, where $n$ is the bit-vector size. We experimented with $*$ BMDs for verification of our applications, and found that $*$ BMDs also do not perform satisfactorily. Note that in our applications, not only do we need to construct *BMDs for higher-degree terms, the word-lengths of the vectors are also different. These finite word-lengths can distort the *BMD structure, which is explained below.

Consider the computation $F[3: 0]=X[1: 0] * Y[1: 0]$. The *BMDs for $F$ is shown in Fig. 5. Now consider $F[1$ : $0]=X[1: 0] * Y[1: 0]$, a fixed-size 2-bit multiplier. The corresponding *BMD structure is shown in Fig. 6. Note that the regular structure of $* \mathrm{BMD}$ is disrupted due to the fixed bit-vector sizes.

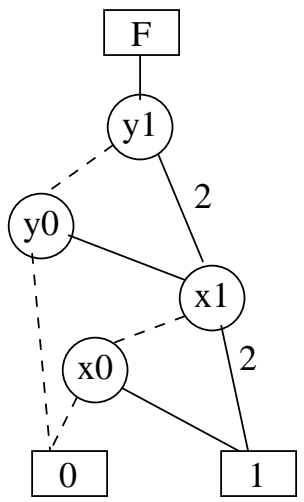

Fig. 5. *BMD for $F=x * y ; x, y$ are 2-bit wide, $F$ is 4-bits wide.

For our experiments, we used the concepts presented in [39] to construct *BMDs directly from the RTL for given wordlengths. In [39], operations for bit-vector manipulations were 
TABLE I

COMPARISON OF TIME TAKEN BY VARIOUS APPROACHES

\begin{tabular}{|c|c|c|c|c|c|}
\hline Benchmark & Specs & Our approach & BDDs-VIS & $\overline{\text { BMD }}$ & SAT-ZChaff \\
\hline & $\operatorname{Var} / \mathbf{D e g} /<n_{1}, \ldots, n_{d}>/ m$ & Time (s) & Nodes/Time(s) & Nodes/Time(s) & Vars/Clauses/Time(s) \\
\hline \multicolumn{6}{|l|}{ Univariate } \\
\hline Anti-alias function & $1 / 6 /<16>/ 16$ & 1.1 & $1.2 \mathrm{M} / 34.21$ & $9 \mathrm{~K} / 96.48$ & $47 \mathrm{~K} / 142 \mathrm{~K} />1000$ \\
\hline Poly_unopt & $1 / 4 /<16>/ 16$ & 0.59 & $0.5 \mathrm{M} / 9.1$ & $1.8 \mathrm{~K} / 14$ & $10 \mathrm{~K} / 28 \mathrm{~K} />1000$ \\
\hline $\cos x$ & $1 / 6 /<32>/ 32$ & 0.59 & $\mathrm{NA} />1000$ & $\mathrm{NA} />1000$ & $60 \mathrm{~K} / 173 \mathrm{~K} />1000$ \\
\hline $\cot ^{-1} x$ & $1 / 9 /<32>/ 32$ & 0.83 & $\mathrm{NA} />1000$ & $\mathrm{NA} />1000$ & $140 \mathrm{~K} / 406 \mathrm{~K} />1000$ \\
\hline $\operatorname{erf} x$ & $1 / 7 /<32>/ 32$ & 0.81 & $\mathrm{NA} />1000$ & $\mathrm{NA} />1000$ & $88 \mathrm{~K} / 255 \mathrm{~K} />1000$ \\
\hline $\ln \left(\frac{1+x}{1-x}\right)$ & $1 / 7 /<32>/ 32$ & 0.55 & $\mathrm{NA} />1000$ & $\mathrm{NA} />1000$ & $86 \mathrm{~K} / 247 \mathrm{~K} />1000$ \\
\hline Vanishing polynomial & $1 / 10 /<8>/ 8$ & 2.18 & $\mathrm{NA} />1000$ & $\mathrm{NA} />1000$ & -NA- \\
\hline \multicolumn{6}{|l|}{ Multivariate } \\
\hline IRR & $2 / 4 /<12,8>/ 16$ & 2.13 & $\mathrm{NA} />1000$ & $1.5 \mathrm{~K} / 13.9$ & $10 \mathrm{~K} / 30 \mathrm{~K} />1000$ \\
\hline PSK & $2 / 4 /<11,14>/ 16$ & 1.07 & $\mathrm{NA} />1000$ & $14.4 \mathrm{~K} / 126.3$ & $61 \mathrm{~K} / 183 \mathrm{~K} />1000$ \\
\hline Cubic filter & $3 / 3 /<24,28,31>/ 32$ & 1.14 & $\mathrm{NA} />1000$ & $\mathrm{NA} />1000$ & $120 \mathrm{~K} / 366 \mathrm{~K} />1000$ \\
\hline Degree-4 filter & $3 / 4 /<15,11,13>/ 16$ & 1.89 & $\mathrm{NA} />1000$ & $\mathrm{NA} />1000$ & $69 \mathrm{~K} / 205 \mathrm{~K} />1000$ \\
\hline Savitzky-Golay filter & $5 / 3 /<16,16,14,12,8>/ 16$ & 1.17 & $\mathrm{NA} />1000$ & $\mathrm{NA} />1000$ & $64 \mathrm{~K} / 190 \mathrm{~K} />1000$ \\
\hline $4^{\text {th }}$ Order IIR & $2 / 4 /<24,29>/ 32$ & 1.39 & $\mathrm{NA} />1000$ & $\mathrm{NA} />1000$ & $214 \mathrm{~K} / 647 \mathrm{~K} />1000$ \\
\hline MIBENCH & $2 / 9 /<16,12>/ 16$ & 4.85 & $26 \mathrm{M} / 16.7$ & $\mathrm{NA} />1000$ & $24 \mathrm{~K} / 69 \mathrm{~K} />1000$ \\
\hline Horner Polynomial 1 & $3 / 4 /<14,14,16>/ 16$ & 1.18 & $\mathrm{NA} />1000$ & $3.3 \mathrm{~K} / 35.2$ & $12 \mathrm{~K} / 37 \mathrm{~K} />1000$ \\
\hline Horner Polynomial 2 & $2 / 4 /<13,13>/ 16$ & 0.62 & $\mathrm{NA} />1000$ & $5.4 \mathrm{~K} / 34.4$ & $23 \mathrm{~K} / 70 \mathrm{~K} />1000$ \\
\hline Vanishing polynomial & $2 / 10 /<12,12>/ 16$ & 4.2 & $\mathrm{NA} />1000$ & $\mathrm{NA} />1000$ & $10 \mathrm{~K} / 29 \mathrm{~K} />1000$ \\
\hline
\end{tabular}

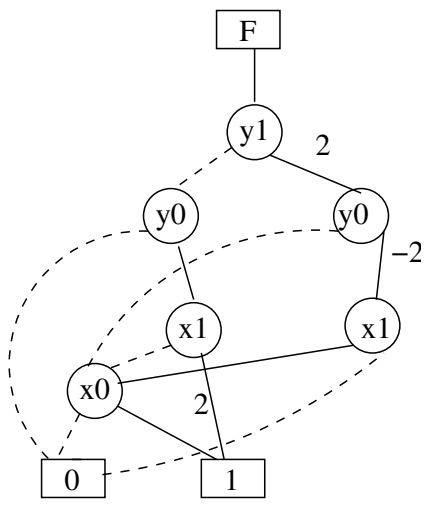

Fig. 6. *BMD for $F=x * y ; x, y, F$ are all 2-bits wide.

described that allow to perform bit-field extraction directly from a given BMD. Using these concepts, *BMDs were constructed directly from the DFGs obtained for the corresponding RTL. *BMD computation terminated only for upto degree-4 polynomials, and the rest of the designs could not be verified within the time-limit.

\section{A. Faulty Designs}

We also wanted to analyze the performance of our approach in the presence of bugs. To verify that our algorithm can detect non-equivalence of designs, we experimented with some designs by arbitrarily changing one or more of the coefficients. Table II presents the results for some of the benchmarks. The algorithm was indeed able to verify that the designs were not equivalent, and that too very quickly. This result is not surprising. The algorithm may not always have to perform $S F\left(2^{m}\right)$ iterations - if the conditions on the coefficients (Theorem 1 and 2) are not satisfied in any iteration (i.e. a bug is found), the algorithm terminates.

We also experimented with simulation and SAT tools to evaluate their performance on detecting non-equivalence. For
TABLE II

NON-EQUIVALENCE DETECTION EXPERIMENTS

\begin{tabular}{||l|c|c|c||}
\hline Benchmark & Our approach & Simulation & SAT \\
\hline \hline Anti-alias function & $0.53 \mathrm{sec}$ & 12 vectors & $2.54 \mathrm{sec}$ \\
\hline Degree-4 filter 1 & $0.57 \mathrm{sec}$ & 8 vectors & $0.59 \mathrm{sec}$ \\
\hline PSK & $0.49 \mathrm{sec}$ & 14 vectors & $1.97 \mathrm{sec}$ \\
\hline Cubic filter & $0.58 \mathrm{sec}$ & 5 vectors & $0.31 \mathrm{sec}$ \\
\hline $4^{t h}$ Order IIR & $0.55 \mathrm{sec}$ & 9 vectors & $0.78 \mathrm{sec}$ \\
\hline
\end{tabular}

simulation, the vectors were generated (pseudo-)randomly, and were applied to both original and faulty RTLs. Nonequivalence was indeed detected within very few vectors. The results are shown in the third column in Table II. For experiments with SAT, we synthesized both original and modified (faulty) designs into gate-level netlists, generated corresponding miter circuits and converted them into CNF format. The ZChaff tool was then used to find a satisfying assignment, thereby proving non-equivalence. Indeed, nonequivalence was easily detected using SAT too, the run time is depicted in the fourth column in Table II. The results show that our technique is comparable against simulation and SAT for detecting non-equivalence of designs.

\section{B. Limitations of our Approach}

Many DSP systems implement some form of computation approximation, by incorporating various rounding schemes. Our approach is restricted inasmuch it cannot verify datapaths where the intermediate signals have varying precision (due to rounding). In such situations, the varying word-length paradigm cannot be easily captured by formulating it as an ideal membership testing instance.

In addition to truncation and rounding, saturation arithmetic is also a common mode of arithmetic approximation in the DSP domain. Traditionally, it has been difficult to model such descriptions as polynomials due to the presence of comparison 
operations. However, when the word-lengths are fixed, it might be possible in some cases to abstract them as polyfunctions. For example, consider the computation:

if $(x>2)$ then $y=x * x * x$ else $y=x * x$;

where $x$ is a 2-bit number and $y$ is 5-bits wide. The 5-bit output $y$ can be represented as a polyfunction from $Z_{2^{2}} \rightarrow Z_{2^{5}}$ as $Y=\left(3 x^{3}+8 x^{2}+22 x\right) \% 2^{5}$. Moreover, arithmetic datapaths often contain right-shift operations which cannot be easily modeled in our framework. Analysis of such computations requires substantially more work, and is the subject of our future investigations.

\section{CONCLUSIONS}

We have presented a framework for equivalence verification of arithmetic datapaths wherein the operands have finite, prespecified word-lengths. Our approach models the design as a polyfunction from $Z_{2^{n_{1}}} \times Z_{2^{n_{2}}} \times \ldots \times Z_{2^{n_{d}}} \rightarrow Z_{2^{m}}$. Subsequently, to prove the equivalence of two designs, we transform the problem $F \% 2^{m} \equiv G \% 2^{m}$ into that of proving $F-G \equiv 0 \% 2^{m}$ over such mappings. This formulation corresponds to that of testing for membership in the ideal of all vanishing polynomials over the given finite ring. Such vanishing ideals have been analyzed and we have derived efficient algorithmic approaches to test whether or not a polynomial is a member of this vanishing ideal. Using our algorithms, a variety of benchmarks have been verified. Our approach was able to solve the problem in all cases, where contemporary verification approaches were shown to be infeasible. As part of future work, we are investigating applications of the proposed concepts to datapath computations that implement other modes of arithmetic approximations, such as rounding and saturation.

\section{REFERENCES}

[1] V. J. Mathews and G. L. Sicuranza, Polynomial Signal Processing. Wiley-Interscience, 2000

[2] R. J. B. T. Allenby, Rings, Fields, and Groups: An Introduction to Abstract Algebra. E. J. Arnold, 1983.

[3] D. Menard, D. Chillet, F. Charot, and O. Sentieys, "Automatic Floatingpoint to Fixed-point Conversion for DSP Code Generation," in Intl. Conf. Compiler, Architecture, Synthesis Embedded Sys., CASES, 2002.

[4] I. A. Groute and K. Keane, "M(VH)DL: A MATLAB to VHDL Conversion Toolbox for Digital Control," in IFAC Symp. on ComputerAided Control System Design, Sept. 2000.

[5] "Simulink: Matlab to rtl translator," http://www.mathworks.com/products/simulink.

[6] A. Peymandoust and G. DeMicheli, "Application of Symbolic Computer Algebra in High-Level Data-Flow Synthesis," IEEE Trans. CAD, vol. 22, no. 9, pp. 1154-11656, 2003.

[7] A. Hosangadi, F. Fallah, and R. Kastner, "Factoring and eliminating common subexpressions in polynomial expressions," in ICCAD, 2004, pp. 169-174.

[8] S. Gopalakrishnan, P. Kalla, and F. Enescu, "Optimizing Fixed-Size BitVector Arithmetic using Finite-Ring Algebra," in International Workship on Logic and Synthesis, 2006.

[9] K. Kum and W. Sung, "Word-length optimization for high-level synthesis of DSP systems," in Intl. workshop Signal Processing Systems, SIPS, 1998.

[10] Z. Chen, "On polynomial functions from $\mathrm{Z}_{n_{1}} \times \mathrm{Z}_{n_{2}} \times \cdots \times \mathrm{Z}_{n_{r}}$ to $\mathrm{Z}_{m}$," Discrete Math., vol. 162, no. 1-3, pp. 67-76, 1996.

[11] O. H. Ibarra and S. Moran, "Probabilistic Algorithms for Deciding Equivalence of Straight-Line Programs," Journal of the Association for Computing Machinery, vol. 30, no. 1, pp. 217-228, Jan. 1983.
[12] I. Niven and J. L. Warren, "A Generalization of Fermat's Theorem," Proc. of American Mathematical Society, vol. 8, pp. 306-313, 1957.

[13] D. Singmaster, "On Polynomial Functions (mod m)," J. Number Theory, vol. 6, pp. 345-352, 1974.

[14] Z. Chen, "On polynomial functions from $Z_{n}$ to $Z_{m}$," Discrete Math. vol. 137, no. 1-3, pp. 137-145, 1995.

[15] R. E. Bryant, "Graph Based Algorithms for Boolean Function Manipulation," IEEE Trans. on Computers, vol. C-35, pp. 677-691, August 1986.

[16] R. E. Bryant and Y.-A. Chen, "Verification of Arithmetic Functions with Binary Moment Diagrams," in $D A C, 95$.

[17] S. Horeth and Drechsler, "Formal Verification of Word-Level Specifications," in DATE, 1999, pp. 52-58.

[18] M. Ciesielski, P. Kalla, Z. Zheng, and B. Rouzyere, "Taylor Expansion Diagrams: A Compact Canonical Representation with Applications to Symbolic Verification," in Proc. Design Automation and Test in Europe, DATE'02, Mar 2002.

[19] C. W. Barlett, D. L. Dill, and J. R. Levitt, "A Decision Procedure for bit-Vector Arithmetic," in DAC, June 1998.

[20] Z. Zhou and W. Burleson, "Equivalence Checking of Datapaths Based on Canonical Arithmetic Expressions," in DAC, 95.

[21] R. Brinkmann and R. Drechsler, "RTL-Datapath Verification using Integer Linear Programming," in Proc. ASP-DAC, 2002.

[22] B. Akbarpour and S. Tahar, "Verification of FFT using HOL Theorem Proving," Concordia Univ., Tech. Rep., 2004.

[23] V. Paruthi, N. Mansouri, and R. Vemuri, "Automatic datapath abstraction for verification of large scale designs," in Intl. Conf. on Computer Design, 1998.

[24] D. Pradhan, "A Theory of Galois Switching Functions," IEEE Trans. on Computers, vol. C-27, no. 3, pp. 239-248, 1978.

[25] K. Ravi and et al., "Modular Verification of Multipliers," in Formal Methods in CAD, 1996.

[26] J. Harrison and L. Thery, "Extending HOL theorem prover with a computer algebra system to reason about the reals," in Proc. Higher Order Logic Theorem Proving and its Applications, 1994.

[27] J. Smith and G. DeMicheli, "Polynomial methods for component maching and verification," in In Proc. ICCAD, 1998.

[28] B. Buchberger, "Ein Algorithmus zum Auffinden der Basiselemente des Restklassenringes nach einem nulldimensionalen Polynomideal," Ph.D. dissertation, Philosophiesche Fakultat an der Leopold-FranzensUniversitat, Austria, 1965.

[29] Maple, http://www.maplesoft.com.

[30] S. Farr and S. Gao, "Computing Gröbner Bases for Vanishing Ideals of Finite Sets of Points," in In Conference on the Applications of Computer Algebra (ACA), 2003.

[31] J. Grabmeier, E. Kaltofen, and V. Weispfenning, Computer Algebra Handbook. Springer-Verlag, 2003.

[32] F. Smarandache, "A function in number theory," Analele Univ. Timisoara, Fascicle 1, vol. XVII, pp. 79-88, 1980.

[33] A. J. Kempner, "Miscellanea." Amer. Math. Monthly, vol. 25, pp. 201$210,1918$.

[34] A. K. Verma and P. Ienne, "Improved use of the Carry-save Representation for the Synthesis of Complex Arithmetic Circuits," in Proceedings of the International Conference on Computer Aided Design, 2004.

[35] M. R. Guthaus, J. S. Ringenberg, D. Ernst, T. M. Austin, T. Mudge, and R. B. Brown, "Mibench: A Free, Commercially Representative Embedded Benchmark Suite," in IEEE 4th Annual Workshop on Workload Characterization, Dec 2001.

[36] U. de Bretagne Sud LESTER, "Gaut, Architectural Synthesis Tool," http://lester.univ-ubs.fr:8080, 2004.

[37] R. K. Brayton, G. D. Hachtel, A. Sangiovanni-Vencentelli, F. Somenzi, A. Aziz, S.-T. Cheng, S. Edwards, S. Khatri, Y. Kukimoto, A. Pardo, S. Qadeer, R. Ranjan, S. Sarwary, G. Shiple, S. Swamy, and T. Villa, "VIS: A System for Verification and Synthesis," in Computer aided Verifi cation, 1996.

[38] M. Moskewicz, C. Madigan, L. Zhao, and S. Malik, "CHAFF: Engineering an Efficient SAT Solver," in In Proc. Design Automation Conference, June 2001, pp. 530-535.

[39] L. Arditi, "*BMDs can Delay the use of Theorem Proving for Verifying Arithmetic Assembly Instructions," in In Proc. Formal methods in CAD, Srivas, Ed. Springer-Verlag, 1996. 
Namrata Shekhar Namrata Shekhar received the B.E. degree in electrical and electronics engineering from the Birla Institute of Technology and Science, Pilani, India in 2002 and is currently working to-

PLACE PHOTO HERE

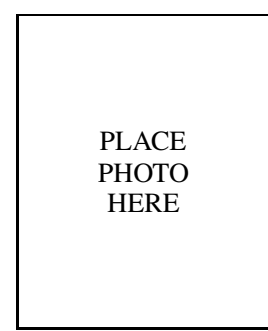

Priyank Kalla Priyank Kalla received the B.E. degree in Electronics Engineering from the Birla Vishvakarma Mahavidyalaya, Saradar Patel University in India in 1993, and M.S. and PhD. degrees from University of Massachusetts Amherst in 1998 and 2002, respectively. He has been an Assistant Professor in the Electrical and Computer Engineering Department, University of Utah, Salt Lake City, since 2002. His research interests are in fundamental CAD techniques for synthesis, optimization, and verification of digital VLSI circuits and systems. Dr.

Kalla is a recipient of the NSF CAREER award in 2006.

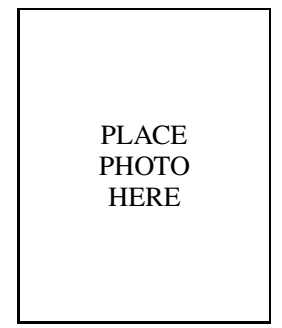

Florian Enescu Florian Enescu has received his BS degree in mathematics from the University of Bucharest, Romania in 1996. While on leave from the Institute of Mathematics of the Romanian Academy of Sciences, he continued his graduate studies in mathematics at the University of Michigan at Ann Arbor where he obtained his MS and $\mathrm{PhD}$ degrees in 1999, respectively 2001. From 2001 to 2004 he was a Scott Assistant Professor of Mathematics at the University of Utah. In Fall 2002, he was a postdoctoral fellow at the Mathematical Sciences Research Institute in Berkeley, California. He has been an assistant professor in the Department of Mathematics and Statistics at Georgia State University, Atlanta, GA since 2004. His research is in commutative algebra, primarily positive characteristic methods in ring theory. He is a member of the American Mathematical Society and the Mathematical Association of America. 